\title{
Estimating heights using auxiliary functions
}

by

\author{
Charles L. Samuels (Bonn)
}

1. Introduction. Let $K$ be a number field and let $v$ be a place of $K$ dividing the place $p$ of $\mathbb{Q}$. Of course, if $v$ is non-Archimedean then $p$ is a rational prime while if $v$ is Archimedean then $p=\infty$. We write $K_{v}$ to denote the completion of $K$ at $v$ and $\mathbb{Q}_{p}$ to denote the completion of $\mathbb{Q}$ at $p$. It is clear that these completions do not depend on a specific absolute value taken from the places $v$ and $p$. We write $d=[K: \mathbb{Q}]$ for the global degree and $d_{v}=\left[K_{v}: \mathbb{Q}_{p}\right]$ for the local degree.

We now select two absolute values on $K_{v}$ for each place $v$. The first absolute value, denoted $\|\cdot\|_{v}$, is the unique extension of the $p$-adic absolute value on $\mathbb{Q}_{p}$. The second, denoted $|\cdot|_{v}$, is defined by

$$
|x|_{v}=\|x\|_{v}^{d_{v} / d}
$$

for all $x \in K_{v}$. We note the important identity

$$
d=\sum_{v \mid p} d_{v}
$$

as well as the product formula

$$
\prod_{v}|\alpha|_{v}=1
$$

for all $\alpha \in K^{\times}$. Furthermore, each of the above absolute values extends uniquely to an algebraic closure $\bar{K}_{v}$. If $v$ is Archimedean then $\bar{K}_{v}$ is complete, however, in general, $\bar{K}_{v}$ is not complete and we write $\Omega_{v}$ to denote its completion. It is well-known that $\Omega_{v}$ is algebraically closed for all places $v$. Moreover, we may define the Weil height of $\alpha \in K$ by

$$
h(\alpha)=\prod_{v} \max \left\{1,|\alpha|_{v}\right\}
$$

2000 Mathematics Subject Classification: Primary 11R04, 11R09.

Key words and phrases: projective height, Weil height, subspace height, Mahler measure, Lehmer's problem. 
where the product is taken over all places $v$ of $K$. By the way we have normalized our absolute values, this definition does not depend on $K$, and therefore we get a well-defined function on $\overline{\mathbb{Q}}$.

For $f \in \mathbb{Z}[x]$ with roots $\alpha_{1}, \ldots, \alpha_{d}$ we define the Mahler measure of $f$ by

$$
\mu(f)=\prod_{k=1}^{d} h\left(\alpha_{k}\right) .
$$

Since $h$ is invariant under Galois conjugation over $\mathbb{Q}$, we note that if $f$ is irreducible and $\alpha$ is any root of $f$ then $\mu(f)=h(\alpha)^{\operatorname{deg} \alpha}$.

By Kronecker's theorem, $\mu(f) \geq 1$ with equality precisely when $f$ is a product of cyclotomic polynomials and $\pm x$. Further, in 1933, D. H. Lehmer [4] asked if there exists a constant $c>1$ such that $\mu(f) \geq c$ in all other cases. It can be computed that

$$
\mu\left(x^{10}+x^{9}-x^{7}-x^{6}-x^{5}-x^{4}-x^{3}+x+1\right)=1.17 \ldots,
$$

which remains the smallest known Mahler measure greater than 1 .

Since Lehmer's famous 1933 paper, many special cases of his proposed problem have been solved. In 1971, Smyth [7] showed that if $\alpha$ and $\alpha^{-1}$ are not Galois conjugates, then the minimal polynomial of $\alpha$ over $\mathbb{Q}$ has Mahler measure at least $\mu\left(x^{3}-x-1\right)$. In a different direction, Schinzel [6] showed as a corollary to a more general result that if $f \in \mathbb{Q}[x]$ has only real roots then $\mu(f) \geq(1+\sqrt{5}) / 2$.

Recently, Borwein, Dobrowolski and Mossinghoff [1] showed that if $f \in$ $\mathbb{Z}[x]$ has no cyclotomic factors and has coefficients congruent to 1 modulo an integer $m$, then

$$
\mu(f) \geq c_{m}(T)^{(\operatorname{deg} f) /(1+\operatorname{deg} f)} .
$$

Here, $c_{m}(T)>1$ is a constant depending on $m$ and an auxiliary polynomial $T \in \mathbb{Z}[x]$. They were able to obtain an explicit lower bound for $\mu(f)$ by making a choice of auxiliary polynomial T. Moreover, Dubickas and Mossinghoff [2] generalized the results of [1] so that the polynomial $f$ in (1.1) may be any factor of a polynomial having coefficients congruent to 1 modulo $m$. They also constructed a sequence of auxiliary polynomials that further improved the explicit bounds given in [1]. Following these methods, the author [5] constructed a function $U(\alpha, T)$ and showed that

$$
1=h(\alpha) \cdot U(\alpha, T)
$$

for all polynomials $T$ over $\overline{\mathbb{Q}}$ with $T(\alpha) \neq 0$. We now briefly recall this construction.

Define the local supremum norm of $T \in \Omega_{v}[x]$ on the unit ball by

$$
\nu_{v}(T)=\sup \left\{|T(z)|_{v}: z \in \Omega_{v} \text { and }|z|_{v} \leq 1\right\} .
$$


Consider the vector space $\mathcal{J}_{v}$ of polynomials over $\Omega_{v}$ of degree at most $N-1$. For $\alpha \in \Omega_{v}$ and $T \in \mathcal{J}_{v}$ define

$$
U_{v}(\alpha, T)=\inf \left\{\nu_{v}(T-f): f \in \mathcal{J}_{v} \text { and } f(\alpha)=0\right\} .
$$

Lemma 2.1 of [5] states that

$$
|T(\alpha)|_{v}=\max \left\{1,|\alpha|_{v}\right\}^{N} \cdot U_{v}(\alpha, T) .
$$

Now assume that $\alpha \in \overline{\mathbb{Q}}$ and $T \in \overline{\mathbb{Q}}[x]$ with $T(\alpha) \neq 0$. In this situation, we may define a global version of $U_{v}(\alpha, T)$ by

$$
U(\alpha, T)=\prod_{v} U_{v}(\alpha, T)
$$

where the product runs over all places $v$ of a number field containing $\alpha$ and the coefficients of $T$. According to (1.5), this product is indeed finite and does not depend on this number field. We may apply the product formula to $|T(\alpha)|_{v}$ to obtain (1.2).

The advantage of this identity is that we may freely select $T$ in a way that is convenient without changing the value of $U(\alpha, T)$. It can then be used to estimate the Weil height in certain special cases as found in [5]. Our goal for the present paper is to apply this strategy to obtain analogous results regarding the projective height and, more generally, the height on subspaces.

If $\mathbf{a}=\left(a_{1}, \ldots, a_{N}\right) \in \Omega_{v}^{N}$, define the local projective height of $\mathbf{a}$ by

$$
H_{v}(\mathbf{a})=\max \left\{\left|a_{1}\right|_{v}, \ldots,\left|a_{N}\right|_{v}\right\} .
$$

That is, the local projective height is simply the maximum norm on $\Omega_{v}^{N}$ with respect to $|\cdot|_{v}$. It is worth noting that some authors define the projective height using the maximum norm only at the non-Archimedean places while using the $L^{2}$ norm on the components of $\mathbf{a}$ at the Archimedean places. However, we are motivated by generalizing the Weil height, so we will find it more relevant to use the maximum norm at all places in our definition. Indeed, we note that

$$
H\left(\left(1, \alpha, \ldots, \alpha^{N}\right)\right)=h(\alpha)^{N} .
$$

It is clear that $H_{v}(\mathbf{a})=1$ for almost all places $v$ of $K$, so we may define the global projective height of $\mathbf{a} \in K^{N}$ by

$$
H(\mathbf{a})=\prod_{v} H_{v}(\mathbf{a})
$$

where the product is taken over all places $v$ of $K$. Of course, by the way we have chosen our absolute values, this definition does not depend on $K$. Furthermore, the product formula implies that $H(\mathbf{a})$ is well defined on $\mathbb{P}^{N-1}(\overline{\mathbb{Q}})$. In Section 2, we define $U(\mathbf{a}, T)$ analogously to (1.4) and prove that

$$
1=H(\mathbf{a})^{M} \cdot U(\mathbf{a}, T) .
$$


Here $T$ is a homogeneous polynomial of degree $M$ in $N$ variables over $\overline{\mathbb{Q}}$ with $T(\mathbf{a}) \neq 0$. We also give a simple example of this result to demonstrate how it might be applied.

In a slightly different direction, suppose that $W$ is a subspace of $\overline{\mathbb{Q}}^{N}$ with basis $\left\{\mathbf{w}_{1}, \ldots, \mathbf{w}_{N}\right\}$. The height of $W$ is defined to be the height of the vector $\mathbf{w}_{1} \wedge \cdots \wedge \mathbf{w}_{M}$ in the exterior product $\bigwedge^{M}\left(\overline{\mathbb{Q}}^{N}\right)$. That is,

$$
H(W)=H\left(\mathbf{w}_{1} \wedge \cdots \wedge \mathbf{w}_{M}\right) .
$$

This definition does not depend on $K$, and it follows from the product formula that $H(W)$ does not depend on our choice of basis. In Section 3, we define $U(W, \Psi)$ for a surjective linear transformation $\Psi: \overline{\mathbb{Q}}^{N} \rightarrow \overline{\mathbb{Q}}^{M}$ and prove that

$$
1=H(W) \cdot U(W, \Psi)
$$

whenever $W \cap \operatorname{ker} \Psi=\{\mathbf{0}\}$. This provides an analog of (1.2) using the height on subspaces.

2. The projective height using auxiliary homogeneous polynomials. We begin by defining the function $U(\mathbf{a}, T)$ given in (1.7). Let $\mathcal{L}_{v}$ denote the vector space of homogeneous polynomials over $\Omega_{v}$ of degree $M$ in $N$ variables along with the zero polynomial. We define an analog of the local supremum norm on polynomials by

$$
\nu_{v}(T)=\sup \left\{|T(\mathbf{z})|_{v}: \mathbf{z} \in \Omega_{v}^{M}, H_{v}(\mathbf{z}) \leq 1\right\}
$$

and set

$$
U_{v}(\mathbf{a}, T)=\inf \left\{\nu_{v}(T-f): f \in \mathcal{L}_{v}, f(\mathbf{a})=0\right\}
$$

for $T \in \mathcal{L}_{v}$. This is the local version of $U(\mathbf{a}, T)$ that will appear in our theorem. Let

$$
Z(\mathbf{a})=\left\{f \in \mathcal{L}_{v}: f(\mathbf{a})=0\right\} .
$$

It is obvious that (2.2) descends to a norm on the one-dimensional quotient $\mathcal{L}_{v} / Z(\mathbf{a})$, so that the ratio $|T(\mathbf{a})|_{v} / U_{v}(\mathbf{a}, T)$ does not depend on $T$. In fact, we are able to prove something much stronger.

Lemma 2.1. If $\mathbf{a} \in \Omega_{v}^{N}$ then

$$
|T(\mathbf{a})|_{v}=H_{v}(\mathbf{a})^{M} \cdot U_{v}(\mathbf{a}, T) \quad \text { for all } T \in \mathcal{L}_{v} .
$$

Proof. We assume that $\left|a_{n}\right|_{v}=H_{v}(\mathbf{a})$ and note that

$$
|T(\mathbf{a})|_{v}=\left|a_{n}\right|_{v}^{M} \cdot\left|T\left(\frac{\mathbf{a}}{a_{n}}\right)\right|_{v} \leq H_{v}(\mathbf{a})^{M} \cdot \nu_{v}(T)
$$


for all homogeneous polynomials $T$ of degree $M$ in $N$ variables. Therefore if $f(\mathbf{a})=0$ then

$$
|T(\mathbf{a})|_{v}=|T(\mathbf{a})-f(\mathbf{a})|_{v} \leq H_{v}(\mathbf{a}) \cdot \nu_{v}(T-f) .
$$

Taking the infimum of the right hand side of (2.4) over all $f$ with $f(\mathbf{a})=0$ we obtain

$$
|T(\mathbf{a})|_{v} \leq H_{v}(\mathbf{a}) \cdot U_{v}(\mathbf{a}, T) .
$$

We now attempt to establish the opposite inequality. We have

$$
\begin{aligned}
U_{v}(\mathbf{a}, T) & =\inf \left\{\nu_{v}(T-f): f \in Z(\mathbf{a})\right\} \\
& =\inf \left\{\nu_{v}\left(T(\mathbf{z})-\left(T(\mathbf{z})-T(\mathbf{a})\left(z_{n} / a_{n}\right)^{M}\right)-T(\mathbf{a}) f(\mathbf{z})\right): f \in Z(\mathbf{a})\right\} \\
& =\inf \left\{\nu_{v}\left(T(\mathbf{a})\left(z_{n} / a_{n}\right)^{M}-T(\mathbf{a}) f(\mathbf{z})\right): f \in Z(\mathbf{a})\right\} \\
& =|T(\mathbf{a})|_{v} \cdot U_{v}\left(\mathbf{a},\left(z_{n} / a_{n}\right)^{M}\right) .
\end{aligned}
$$

It is clear that

$$
U_{v}\left(\mathbf{a},\left(z_{n} / a_{n}\right)^{M}\right) \leq \nu_{v}\left(\left(z_{n} / a_{n}\right)^{M}\right)=\sup \left\{\left|z_{n} / a_{n}\right|_{v}^{M}:\left|z_{n}\right| \leq 1\right\}=\left|a_{n}\right|_{v}^{-M}
$$

and hence

$$
U_{v}(\mathbf{a}, T) \leq|T(\mathbf{a})|_{v} \cdot H_{v}(\mathbf{a})^{-M},
$$

which completes the proof.

If $T$ is a homogeneous polynomial over $K$ of degree $M$ in $N$ variables and $\mathbf{a} \in K^{N}$ then Lemma 2.1 implies that $\nu_{v}(\mathbf{a}, T)=1$ for almost all places $v$ of $K$. Hence, we may define the global functions

$$
\nu(T)=\prod_{v} \nu_{v}(T) \text { and } U(\mathbf{a}, T)=\prod_{v} U_{v}(\mathbf{a}, T),
$$

which do not depend on $K$. We now obtain the following projective generalization of (1.2).

Theorem 2.2. If $\mathbf{a} \in \overline{\mathbb{Q}}^{N}$ then

$$
1=H(\mathbf{a})^{M} \cdot U(\mathbf{a}, T)
$$

for all homogeneous polynomials $T$ over $\overline{\mathbb{Q}}$ of degree $M$ in $N$ variables having $T(\alpha) \neq 0$.

Proof. Suppose that $K$ is a number field containing the entries of a and the coefficients of $T$. Hence, we may view a as an element of $\Omega_{v}^{N}$ and $T$ as an element of $\mathcal{L}_{v}$ for all places $v$ of $K$. Thus, Lemma 2.1 implies that

$$
|T(\mathbf{a})|_{v}=H_{v}(\mathbf{a})^{M} \cdot U_{v}(\mathbf{a}, T)
$$

at every place $v$ of $K$. The result follows by taking the product of (2.7) over all places of $K$ and applying the product formula to $T(\mathbf{a})$.

We may construct applications of Theorem 2.2 that are similar to those found in [5]. Suppose, for example, that $F$ is a homogeneous polynomial 
of degree $M$ in $N$ variables with coefficients in $\mathbb{Z}$. Let $\mathcal{X}(F)$ denote the subvariety of $\mathbb{P}^{N-1}(\overline{\mathbb{Q}})$ consisting of all points a with $F(\mathbf{a})=0$. Suppose further that $T$ homogeneous of degree $M$ in $N$ variables and $m \in \mathbb{Z}$ are such that

$$
T \equiv F \bmod m .
$$

That is, the coefficients of $T$ are congruent to the coefficients of $F$ modulo $m$. If $\mathbf{a} \in \mathcal{X}(T)$ then Theorem 2.2 implies that

$$
1=H(\mathbf{a})^{M} \cdot U(\mathbf{a}, T) .
$$

Now select a number field $K$ containing the entries of $\mathbf{a}$. If $v$ is non-Archimedean then

$$
U_{v}(\mathbf{a}, T) \leq \nu_{v}(T-F) \leq|m|_{v}
$$

so that

$$
U(\mathbf{a}, T) \leq \nu_{\infty}(T) \prod_{v \nmid \infty}|m|_{v}=m^{-1} \cdot \nu_{\infty}(T) .
$$

If $T$ has coefficients $c_{1}, \ldots, c_{R} \in \mathbb{Z}$, define

$$
L_{\infty}^{1}(T)=\left(\sum_{r=1}^{R}\left\|c_{r}\right\|_{v}\right)^{d_{v} / d}
$$

and note that by the triangle inequality we have $\nu_{\infty}(T) \leq L_{\infty}^{1}(T)$. Hence, we obtain a lower bound on the projective height of $\mathbf{a}$ :

$$
H(\mathbf{a})^{\operatorname{deg} F} \geq m / L_{\infty}^{1}(T)
$$

for all $\mathbf{a} \in \mathcal{X}(F) \backslash \mathcal{X}(T)$. Hence, if $L_{\infty}^{1}(T)$ is small relative to $m$ then we obtain a uniform lower bound on $H(\mathbf{a})^{\operatorname{deg} F}$ over all $\mathbf{a} \in \mathcal{X}(F) \backslash \mathcal{X}(T)$. In particular, if $T$ is a monomial having coefficient \pm 1 then (2.8) becomes

$$
H(\mathbf{a})^{\operatorname{deg} F} \geq m,
$$

which is non-trivial for all $m \geq 2$.

\section{The height on subspaces using auxiliary linear transforma-}

tions. We now turn our attention to the height on subspaces and attempt to construct an analog of Theorem 2.2. Suppose that $X$ is an $N$-dimensional vector space over $\Omega_{v}$ and fix a basis $\left\{\mathbf{e}_{1}, \ldots, \mathbf{e}_{N}\right\}$ for $X$. For ease of notation, we identify $X$ with $\Omega_{v}^{N}$ by writing

$$
\mathbf{x}=x_{1} \mathbf{e}_{1}+\cdots+x_{N} \mathbf{e}_{N}=\left(x_{1}, \ldots, x_{N}\right) .
$$

In this way, we obtain the projective height of $\mathbf{x} \in X$ by

$$
H_{v}(\mathbf{x})=\max \left\{\left|x_{1}\right|_{v}, \ldots,\left|x_{M}\right|_{v}\right\} .
$$


Of course, this is a norm on $X$, and therefore, it yields the natural dual norm of an element $\phi \in X^{*}$,

$$
\nu_{v}(\phi)=\sup \left\{|\phi(\mathbf{x})|_{v}: \mathbf{x} \in X, H_{v}(\mathbf{x}) \leq 1\right\} .
$$

Now fix an element $\mathbf{w} \in X$ and let

$$
S^{*}(\mathbf{w})=\left\{\phi \in X^{*}: \phi(\mathbf{w})=0\right\},
$$

so that $S^{*}(\mathbf{w})$ is an $N$-1-dimensional subspace of $X^{*}$. Finally, for $\psi \in X^{*}$ we set

$$
U_{v}(\mathbf{w}, \psi)=\inf \left\{\nu_{v}(\psi-\phi): \phi \in S^{*}(\mathbf{w})\right\} .
$$

We note that this defines a norm on the one-dimensional quotient $X^{*} / S^{*}(\mathbf{w})$. Of course, this implies that the ratio $|\psi(\mathbf{w})|_{v} / U_{v}(\mathbf{w}, \psi)$ depends only on $\mathbf{w}$ and $v$. Analogously to the results of [5] and of Section 2 we are able to determine this ratio precisely.

Lemma 3.1. If $\mathbf{w} \in X$ then

$$
|\psi(\mathbf{w})|_{v}=H_{v}(\mathbf{w}) \cdot U_{v}(\mathbf{w}, \psi) \quad \text { for all } \psi \in X^{*} .
$$

Proof. If $\psi(\mathbf{w})=0$ then both sides of the desired identity equal 0 . Hence, we assume that $\psi(\mathbf{w}) \neq 0$. Let $\mathbf{w}=\left(w_{1}, \ldots, w_{N}\right)$ and select an integer $n$ such that $H_{v}(\mathbf{w})=\left|w_{n}\right|_{v}$. Of course, $w_{n} \neq 0$ and $H_{v}\left(\mathbf{w} / w_{n}\right)=1$, so that we obtain

$$
|\psi(\mathbf{w})|_{v}=\left|w_{n}\right|_{v} \cdot\left|\psi\left(\mathbf{w} / w_{n}\right)\right|_{v} \leq H_{v}(\mathbf{w}) \cdot \nu_{v}(\psi)
$$

for all $\psi \in X^{*}$. Hence, if $\phi \in S^{*}(\mathbf{w})$ then

$$
|\psi(\mathbf{w})|_{v}=|(\psi-\phi)(\mathbf{w})|_{v} \leq H_{v}(\mathbf{w}) \cdot \nu_{v}(\psi-\phi) .
$$

Taking the infimum of the right hand side over all $\phi \in S^{*}(\mathbf{w})$ we obtain

$$
|\psi(\mathbf{w})|_{v} \leq H_{v}(\mathbf{w}) \cdot U_{v}(\mathbf{w}, \psi) .
$$

We now attempt to prove the opposite inequality. We define the map $J: X^{*} \rightarrow X$ by

$$
J(\phi)=\left(\phi\left(\mathbf{e}_{1}\right), \ldots, \phi\left(\mathbf{e}_{N}\right)\right)
$$

and note that $J$ is a vector space isomorphism having the property that $\phi(\mathbf{w})=J(\phi) \cdot \mathbf{w}$ where "." represents the inner product. We now define appropriate bases for $X^{*}$ and $S^{*}(\mathbf{w})$. Let $\mathbf{c}_{n}=\left(0, \ldots, 0, w_{n}^{-1}, 0, \ldots, 0\right)^{T}$ and note that $\mathbf{c}_{n} \cdot \mathbf{w}=1$. For each index $k \neq n$, we define $\mathbf{c}_{k}$ in the following way. If $w_{k} \neq 0$ then we let $\mathbf{c}_{k}$ be the vector having $w_{k}^{-1}$ as the $k$ th entry, $-w_{n}^{-1}$ as the $n$th entry, and zero in all other entries. If $w_{k}=0$ then we let $\mathbf{c}_{k}$ be the vector having 1 as the $k$ th entry and 0 elsewhere. Hence, $\left\{J^{-1}\left(\mathbf{c}_{1}\right), \ldots, J^{-1}\left(\mathbf{c}_{N}\right)\right\}$ forms a basis for $X^{*}$ and

$$
\left\{J^{-1}\left(\mathbf{c}_{1}\right), \ldots, J^{-1}\left(\mathbf{c}_{n-1}\right), J^{-1}\left(\mathbf{c}_{n+1}\right), \ldots, J^{-1}\left(\mathbf{c}_{N}\right)\right\}
$$

forms a basis for $S^{*}(\mathbf{w})$. 
Now write $\psi=\psi_{1} J^{-1}\left(\mathbf{c}_{1}\right)+\cdots+\psi_{N} J^{-1}\left(\mathbf{c}_{N}\right)$ and note that $\psi(\mathbf{w})=\psi_{n}$. Therefore,

$$
\begin{aligned}
U_{v}(\mathbf{w}, \psi) & =\inf \left\{\nu_{v}(\psi-\phi): \phi \in S^{*}(\mathbf{w})\right\} \\
& =\inf \left\{\nu_{v}\left(\psi_{1} J^{-1}\left(\mathbf{c}_{1}\right)+\cdots+\psi_{N} J^{-1}\left(\mathbf{c}_{N}\right)-\phi\right): \phi \in S^{*}(\mathbf{w})\right\} \\
& =\inf \left\{\nu_{v}\left(\psi_{n} J^{-1}\left(\mathbf{c}_{n}\right)-\psi_{n} \phi\right): \phi \in S^{*}(\mathbf{w})\right\}=\left|\psi_{n}\right|_{v} \cdot U_{v}\left(\mathbf{w}, J^{-1}\left(\mathbf{c}_{n}\right)\right) \\
& =|\psi(\mathbf{w})|_{v} \cdot U_{v}\left(\mathbf{w}, J^{-1}\left(\mathbf{c}_{n}\right)\right) .
\end{aligned}
$$

Next, we observe that

$$
\begin{aligned}
U_{v}\left(\mathbf{w}, J^{-1}\left(\mathbf{c}_{n}\right)\right) & \leq \nu_{v}\left(J^{-1}\left(\mathbf{c}_{n}\right)\right)=\sup \left\{\left|c_{n} \cdot \mathbf{z}\right|_{v}: H_{v}(\mathbf{z}) \leq 1\right\} \\
& =\left|w_{n}\right|_{v}^{-1}=H_{v}(\mathbf{w})^{-1} .
\end{aligned}
$$

We have found that

$$
U_{v}(\mathbf{w}, \psi) \leq|\psi(\mathbf{w})|_{v} \cdot H_{v}(\mathbf{w})^{-1}
$$

and the result follows from (3.1).

In order to generalize Lemma 3.1 to include the height on subspaces rather than simply the projective height, we must now consider the $M$ th exterior power $\bigwedge^{M}\left(\Omega_{v}^{N}\right)$. We define the index set

$$
\mathcal{I}_{M}=\{I \subset\{1, \ldots, N\}:|I|=M\} .
$$

If $\left\{\mathbf{e}_{1}, \ldots, \mathbf{e}_{N}\right\}$ is the standard basis for $\Omega_{v}^{N}$, we obtain a natural basis

$$
\left\{\bigwedge_{i \in I} \mathbf{e}_{i}: I \in \mathcal{I}\right\}
$$

for $\bigwedge^{M}\left(\Omega_{v}^{N}\right)$ over $\Omega_{v}$. The height of an element $\mathbf{x} \in \bigwedge^{M}\left(\Omega_{v}^{N}\right)$ is computed using the basis (3.2). For $\phi$ belonging to the dual $\left(\bigwedge^{M}\left(\Omega_{v}^{N}\right)\right)^{*}$, the norm of $\phi$ is given by

$$
\nu_{v}(\phi)=\sup \left\{|\phi(\mathbf{x})|_{v}: \mathbf{x} \in \bigwedge^{M}\left(\Omega_{v}^{N}\right), H_{v}(\mathbf{x}) \leq 1\right\} .
$$

If $\mathbf{w} \in \bigwedge^{M}\left(\Omega_{v}^{N}\right)$ then

$$
U_{v}(\mathbf{w}, \psi)=\inf \left\{\nu_{v}(\psi-\phi): \phi \in\left(\bigwedge^{M}\left(\Omega_{v}^{N}\right)\right)^{*}, \phi(\mathbf{w})=0\right\} .
$$

We also obtain the following lemma showing that a surjective linear transformation $\Psi: \Omega_{v}^{N} \rightarrow \Omega_{v}^{M}$ may be viewed as a map on $\bigwedge^{M}\left(\Omega_{v}^{N}\right)$.

Lemma 3.2. Suppose that $\Psi: \Omega_{v}^{N} \rightarrow \Omega_{v}^{M}$ is a surjective linear transformation. Then there exists a unique linear transformation $\bigwedge^{M}(\Psi): \bigwedge^{M}\left(\Omega_{v}^{N}\right)$ $\rightarrow \Omega_{v}$ such that

for all $\mathbf{w}_{1}, \ldots, \mathbf{w}_{M} \in \Omega_{v}^{N}$.

$$
\bigwedge^{M}(\Psi)\left(\mathbf{w}_{1} \wedge \cdots \wedge \mathbf{w}_{M}\right)=\operatorname{det}\left(\begin{array}{c}
\Psi\left(\mathbf{x}_{1}\right) \\
\vdots \\
\Psi\left(\mathbf{x}_{M}\right)
\end{array}\right)
$$


Proof. Let $\mathcal{M}_{M \times M}\left(\Omega_{v}\right)$ denote the vector space of $M \times M$ matrices with entries in $\Omega_{v}$. We note that $\Psi$ induces a unique $M$-multilinear map $\Psi^{\prime}:\left(\Omega_{v}^{N}\right)^{M} \rightarrow \mathcal{M}_{M \times M}\left(\Omega_{v}\right)$ given by

$$
\Psi^{\prime}\left(\mathbf{w}_{1}, \ldots, \mathbf{w}_{M}\right)=\left(\begin{array}{c}
\Psi\left(\mathbf{x}_{1}\right) \\
\vdots \\
\Psi\left(\mathbf{x}_{M}\right)
\end{array}\right)
$$

Furthermore, it is well-known (see, for example, [3, p. 437]) that the determinant map det $: \mathcal{M}_{M \times M}\left(\Omega_{v}\right) \rightarrow \Omega_{v}$ defines an $M$-multilinear map on the rows of the elements in $\mathcal{M}_{M \times M}\left(\Omega_{v}\right)$. Hence, we conclude that the composition deto $\Psi^{\prime}$ is an $M$-multilinear map from $\left(\Omega_{v}^{N}\right)^{M}$ to $\Omega_{v}$. Moreover, if there exist $i \neq j$ with $\mathbf{w}_{i}=\mathbf{w}_{j}$ then

$$
\operatorname{det} \circ \Psi^{\prime}\left(\mathbf{w}_{1}, \ldots, \mathbf{w}_{M}\right)=0 .
$$

It follows that $\operatorname{det} \circ \Psi^{\prime}$ is, in fact, an alternating $M$-multilinear map.

By the universal property for alternating $M$-tensors, there exists a unique linear transformation $T: \bigwedge^{M}\left(\Omega_{v}^{N}\right) \rightarrow \Omega_{v}$ such that

$$
T \circ \iota=\operatorname{det} \circ \Psi^{\prime}
$$

where $\iota:\left(\Omega_{v}^{N}\right)^{M} \rightarrow \bigwedge^{M}\left(\Omega_{v}^{N}\right)$ is given by

$$
\iota\left(\mathbf{w}_{1}, \ldots, \mathbf{w}_{M}\right)=\mathbf{w}_{1} \wedge \cdots \wedge \mathbf{w}_{M} .
$$

Therefore, we conlude that

$$
\begin{aligned}
T\left(\mathbf{w}_{1} \wedge \cdots \wedge \mathbf{w}_{M}\right) & =T\left(\iota\left(\mathbf{w}_{1}, \ldots, \mathbf{w}_{M}\right)\right)=\operatorname{det}\left(\Psi^{\prime}\left(\mathbf{w}_{1}, \ldots, \mathbf{w}_{M}\right)\right) \\
& =\operatorname{det}\left(\begin{array}{c}
\Psi\left(\mathbf{x}_{1}\right) \\
\vdots \\
\Psi\left(\mathbf{x}_{M}\right)
\end{array}\right)
\end{aligned}
$$

By taking $\bigwedge^{M}(\Psi)=T$ we complete the proof.

We now assume that $W$ is an $M$-dimensional subspace of $\overline{\mathbb{Q}}^{N}$ and $\Psi$ : $\overline{\mathbb{Q}}^{N} \rightarrow \overline{\mathbb{Q}}^{M}$ is a surjective linear transformation. Select a basis $\left\{\mathbf{w}_{1}, \ldots, \mathbf{w}_{M}\right\}$ for $W$ and assume that $K$ is a number field containing the entries of each basis element $\mathbf{w}_{m}$ as well as the entries of $\Psi$. We note that the height of $W$ is given by

$$
H(W)=\prod_{v} H_{v}\left(\mathbf{w}_{1} \wedge \cdots \wedge \mathbf{w}_{M}\right)
$$

where the product is taken over all places $v$ of $K$. As we noted in our introduction, the product formula implies that this definition does not depend 
on the choice of basis for $W$. By Lemma 3.2 we may define

$$
U(W, \Psi)=\prod_{v} U_{v}\left(\mathbf{w}_{1} \wedge \cdots \wedge \mathbf{w}_{M}, \wedge^{M}(\Psi)\right) .
$$

Lemma 3.1 shows that this product is indeed finite and, because of the way we have normalized our absolute values, it does not depend on $K$. As in the height on subspaces, the product formula implies that (3.3) is independent of the basis for $W$ as well. We may now state and prove our main result.

Theorem 3.3. If $W$ is an $M$-dimensional subspace of $\overline{\mathbb{Q}}^{N}$ then

$$
1=H(W) \cdot U(W, \Psi)
$$

for all surjective linear transformations $\Psi: \overline{\mathbb{Q}}^{N} \rightarrow \overline{\mathbb{Q}}^{M}$ with $W \cap \operatorname{ker} \Psi=\{\mathbf{0}\}$.

Proof. Let $\left\{\mathbf{w}_{1}, \ldots, \mathbf{w}_{M}\right\}$ be a basis for $W$ and let $K$ be a number field containing the entries of each basis element $\mathbf{w}_{m}$ and the entries of $\Psi$. Hence, $\mathbf{w}_{m} \in \Omega_{v}^{N}$ and $\Psi: \Omega_{v}^{N} \rightarrow \Omega_{v}^{M}$ for all places $v$ of $K$. Therefore, Lemma 3.1 implies that

$$
\begin{aligned}
\mid \wedge^{M}(\Psi)\left(\mathbf{w}_{1}\right. & \left.\wedge \cdots \wedge \mathbf{w}_{M}\right)\left.\right|_{v} \\
& =H_{v}\left(\mathbf{w}_{1} \wedge \cdots \wedge \mathbf{w}_{M}\right) \cdot U_{v}\left(\mathbf{w}_{1} \wedge \cdots \wedge \mathbf{w}_{M}, \wedge^{M}(\Psi)\right) .
\end{aligned}
$$

By Lemma 3.2 we have

$$
\bigwedge^{M}(\Psi)\left(\mathbf{w}_{1} \wedge \cdots \wedge \mathbf{w}_{M}\right)=\operatorname{det}\left(\begin{array}{c}
\Psi\left(\mathbf{w}_{1}\right) \\
\vdots \\
\Psi\left(\mathbf{w}_{M}\right)
\end{array}\right) .
$$

Since $W \cap \operatorname{ker} \Psi=\{\mathbf{0}\}$ we know that the rows in the above matrix are linearly independent, so that its determinant is non-zero. Hence, the left hand side of (3.4) is non-zero and we may apply the product formula. The desired identity follows immediately.

It is natural to consider the special case of Theorem 3.3 in which $W$ is a one-dimensional subspace spanned by an element $\mathbf{w} \in \overline{\mathbb{Q}}^{N}$. For $\psi \in\left(\overline{\mathbb{Q}}^{N}\right)^{*}$ we define

$$
U(\mathbf{w}, \psi)=\prod_{v} U_{v}(\mathbf{w}, \psi)
$$

and obtain the following corollary.

Corollary 3.4. If $\mathbf{w} \in \overline{\mathbb{Q}}^{N}$ then

$$
1=H(\mathbf{w}) \cdot U(\mathbf{w}, \psi)
$$

for all $\psi \in\left(\overline{\mathbb{Q}}^{N}\right)^{*}$ with $\psi(\mathbf{w}) \neq 0$.

Proof. If $W$ is the one-dimensional subspace spanned by $\mathbf{w}$ then it is easy to see that $H(W)=H(\mathbf{w})$. Furthermore, $\psi: \overline{\mathbb{Q}}^{N} \rightarrow \overline{\mathbb{Q}}$ is a linear 
transformation and $U(W, \psi)=U(\mathbf{w}, \psi)$. Theorem 3.3 yields

$$
1=H(W) \cdot U(W, \psi)
$$

and the result follows immediately.

\section{References}

[1] P. Borwein, E. Dobrowolski and M. J. Mossinghoff, Lehmer's problem for polynomials with odd coefficients, Ann. of Math. 166 (2007), 347-366.

[2] A. Dubickas and M. J. Mossinghoff, Auxiliary polynomials for some problems regarding Mahler's measure, Acta Arith. 119 (2005), 65-79.

[3] D. S. Dummit and R. M. Foote, Abstract Algebra, Wiley, Hoboken, NJ, 2004.

[4] D. H. Lehmer, Factorization of certain cyclotomic functions, Ann. of Math. 34 (1933), 461-479.

[5] C. L. Samuels, The Weil height in terms of an auxiliary polynomial, Acta Arith. 128 (2007), 209-221.

[6] A. Schinzel, On the product of the conjugates outside the unit circle of an algebraic number, ibid. 24 (1973), 385-399; addendum, ibid. 26 (1975), 329-331.

[7] C. J. Smyth, On the product of the conjugates outside the unit circle of an algebraic integer, Bull. London Math. Soc. 3 (1971), 169-175.

Max-Planck-Institut für Mathematik

Vivatsgasse 7

53111 Bonn, Germany

E-mail: csamuels@mpim-bonn.mpg.de

Received on 21.3.2008

and in revised form on 2.12.2008 